\title{
Status quo and innovative approaches for maritime logistics in the age of digitalization: a guest editors' introduction
}

\author{
Leonard Heilig ${ }^{1} \cdot$ Stefan Voß ${ }^{1,2}$
}

Published online: 2 September 2017

(C) Springer Science+Business Media, LLC 2017

Maritime logistics can be regarded as the marriage between maritime transportation, handling and moving cargo between and within seaports, and principles of logistics and supply chain management. The unification of those different concepts aims at addressing evolving demands of customers, improving financial and operational performance, service quality, and environmental performance, growth, and corporate responsibility [6]. In recent years, this emerging discipline has attracted increasing attention in academia and practice. Especially the role of ports has drastically changed from its traditional function, connecting sea and landside by facilitating loading and unloading operations, to an essential part of global logistics networks that manages the flow of cargo and offers value-added logistics services in an efficient, effective, safe, and environmental-friendly manner. In this regard, one of the main challenges is to facilitate the coordination and collaboration among various involved actors, especially to manage activities at the interfaces in different phases of transportation, storage, and other logistics activities. Information systems and dedicated decision support systems manage related information flows and support organizational and inter-organizational decision making. Therefore, they are essential for facilitating integration and achieving the goals of maritime logistics strategies. In general,

Leonard Heilig

leonard.heilig@uni-hamburg.de

Stefan Voß

stefan.voss@uni-hamburg.de

1 Institute of Information Systems (IWI), University of Hamburg, 20146 Hamburg, Germany

2 Escuela de Ingenieria Industrial, Pontificia Universidad Católica de Valparaíso, Valparaíso, Chile information systems already pervade nearly all activities in maritime logistics primarily used to acquire, process, exchange, and analyze data at a rapidly increasing volume and pace by applying advanced technologies and methods.

After decades of digital transformation and automation, in particular in seaport and terminal operations (see, e.g., [2]), there is a growing demand to better utilize available and new data sources. With the increase of data sources comes the need to integrate internal and external information, after checking the reliability, quality, and veracity. Increasing the speed of processing from batch processing to real-time processing, a new door is opened to realize innovative maritime logistics approaches considering contextual and environmental factors in individual, crossfunctional, and inter-organizational activities. As such, the development of cutting-edge information systems and methods fully exploiting gathered information for problemsolving and decision making establishes a basis for realizing innovative maritime logistics strategies and approaches like, for instance, synchromodality and slowsteaming. Despite the growing importance of information systems in solving current and future challenges, little attention has been paid to this topic in the area of maritime logistics. The special issue promotes this line of research by focusing on the status quo and innovative approaches with respect to the collection and utilization of information in maritime logistics.

The special issue includes four articles reflecting the state-of-the-art and providing different innovative approaches to cope with current challenges in maritime logistics. The focus is in particular on the utilization of information systems and decision analytics in ports and maritime shipping, which involves the development and application of information technology, operations research, statistics, simulation, and predictive analytics to support decision 
making and problem-solving. In general, those approaches aim to take organizations to a higher degree of transparency, intelligence, and competitive innovation. Thus, the special issue synthesizes different perspectives and solution approaches on real-world problems in the area of maritime logistics and points out new directions and opportunities for both science and business practice.

The lead-in article of the special issue, entitled "Information systems in seaports: a categorization and overview" [3], introduces the reader to the current use of information systems in modern ports and connects between industry solutions and related scientific approaches. The relationships among information systems and underlying information technologies, referred to as enabling technologies, are reflected through a conceptual framework that classifies and surveys information systems vertically and horizontally according to their scope on port operations. The article shows not only how enabling technologies and information systems are currently aligned with port operations, but also how they can be utilized to address current and future challenges, for example, to compensate physical constraints of ports like inadequate infrastructure, capacity bottlenecks, accessibility issues, and environmental problems. The article emphasizes the role of international standards and further explains the link between new innovative information systems, gathering and processing real-time data on all levels of port operations, and existing decision analytics approaches, for example, with respect to vessel scheduling, berth allocation, and yard planning. In this context, new research opportunities are emphasized, such as with regard to improving the capability of ports to improve the economic and ecological performance, reliability, responsiveness, and quality of port operations. This involves, for example, predictive analytics to better forecast arrival and waiting times as well as the identification of errors and means to dynamically re-schedule activities to avoid/reduce cascading effects having a negative impact on the overall logistics chain. Innovative projects and solutions from industry are considered to emphasize the current interest in developing new applications and business models. However, we see that the gap between such topics in academia, receiving attention from scholars for many decades, and industry solutions is growing. Consequently, it seems that it becomes more important than ever to bidirectionally align and merge science and industry solutions. Overall, the article is the first, to the best of our knowledge, presenting an in-depth and comprehensive overview of the utilization of enabling technologies and information systems in ports. Note that the other papers in the special issue partly refer to the enabling technologies and information systems explained and discussed in this article.

The second article is presented by Cimino et al. [1], entitled "Evaluating the impact of smart technologies on harbor's logistics via BPMN modeling and simulation". The authors propose a simulation-based method for evaluating the impact of using advanced information technologies in port operations based on the business process modeling notation (BPMN). The application of the proposed method is demonstrated by assessing the use of wireless sensor networks and RFID for improving container identification and gate processes, respectively, within a container terminal of the Port of Leghorn (Italy). For this purpose, a BPMN model of terminal activities at the Port of Leghorn is proposed and used for a simulation study. Insights on how terminal activities can be further improved through the use of modern information systems are discussed, in particular at interfaces (e.g., terminal gate), such as in terms of resource availabilty, resource usage, and dwell times of cargo in terminals. As such, the article provides a novel approach to measure and evaluate the impact of applying multiple enabling technologies in an integrated chain of logistics activities by considering several scenarios (including peak hours), instead of assessing their individual impact on certain terminal activities. This integrated view on terminal, i.e., port operations is promoted by the area of maritime logistics and becomes increasingly important for implementing and assessing innovative strategies and approaches involving several parts and actors of global logistics chains.

The third article of the special issue, presented by Hill and Böse [4], is entitled "A decision support system for improved resource planning and truck routing at logistic nodes". The article works on the interface between portrelated logistics nodes and drayage transport activities. A critical assessment and solution approaches in this area are urgently needed as many ports nowadays face huge traffic problems, heavily fluctuating workloads, and other uncertainties having an impact on the efficiency of port operations. While logistics nodes in the port (such as container terminals, empty container depots, etc.) facing issues with regard to an efficient resource allocation (e.g., staff planning) due to a lack of actual data, trucking companies might miss the opportunity to better utilize resources by considering potential waiting times at logistics nodes. In this context, the authors present a conceptual design of a decision support system to inform logistics nodes and trucking companies about forecasted truck arrival times and truck gate waiting times, respectively. The information system is designed to integrate data from different enabling technologies, including OCR gates, and information systems installed at the logistics nodes, such as terminal operating systems. Regarding the forecasting engine, the authors propose a predictive analytics approach by applying a neural network using data from a large maritime empty container depot in Northern Germany. Implications with respect to the applicability of the neural network are 
discussed. In general, applying machine learning techniques to better utilize available sources of information for optimizing port operations becomes increasingly important, especially in times where the amount of available data is continuously growing. This opens new opportunities for research on the interface between machine learning and optimization and might also involve the application of metaheuristics in many cases as a means to find sufficiently good solutions within short computational times. The objectives of a higher visibility and more accurate decision support are further discussed in this article.

The last article of the special issue covers the area of maritime shipping by proposing a decision support system designed for liner shipping fleet repositioning. Shipping fleet repositioning is necessary to adjust liner service networks in order to cope with changing trends like production patterns, cargo volumes, and world economic indicators. As it has a huge impact on the revenues and costs of liner shipping companies, decision support is needed to provide competitive services. The article presented by Müller and Tierney [5], entitled "Decision support and data visualization for liner shipping fleet repositioning," advances a simulated annealing approach for solving the underlying liner shipping fleet repositioning problem (LSFRP) by allowing ad-hoc adjustments during the solution process. The interactivity enables repositioning coordinators to adjust optimization settings including port calls, demands, general information of scenarios (e.g., set of vessels), and simulated annealing settings. The optimization problem is modeled as an arc flow model and briefly explained in the article. The developed simulated annealing algorithm is incorporated into a webbased decision support system that processes and illustrates results using various forms of visualization including different graph representations. Those visualizations of liner shipping services and repositionings are discussed regarding their capability to represent important information for decision makers. This aspect further underlines the need for integrative approaches ranging from data collection, through data analysis (e.g., using machine learning techniques), up to information utilization in form of decision support and allocation/distribution of information to dedicated decision makers through individual channels and representations.

The concepts and innovative approaches presented in this special issue offer new opportunities to advance business practice in maritime logistics. However, financial, organizational, policy-making, and professional factors affecting and challenging real implementation have not been considered in this special issue. Also with respect to future skills and roles of actors and people being involved in maritime logistics, more research is necessary. While the importance of disruptive technology trends, such as with respect to the internet of things, cloud computing, and mobile internet has become apparent in the special issue, more research, combining inter-disciplinary knowledge, is crucial to cope with current and future challenges in maritime logistics. This will involve the adaption, integration, and advancement of current solutions and methods, both in academia and practice. Moreover, the integration with other business models and ecosystems like airports, car sharing systems, and urban environments needs to be assessed from different perspectives. Failing to improve and innovate business processes, business networks, and business models through goal-driven digital innovation and advanced decision support may not only deteriorate the competitiveness of actors in the martime logistics industry, but may also lead to a situation where actors are forced out of the market if they cannot fulfill certain demands of customers and/or regulatory requirements. In this respect, the presented articles in the special issue cover different activities and use methodological approaches and perspectives in order to advance the area of maritime logistics through a more efficient utilization of information for decision support. We hope that the special issue helps to promote future research in this new direction of maritime logistics research.

We acknowledge the authors for their expertise, dedication, and patience, particularly their effort to produce high-quality articles during multiple rounds of peer reviews. Worthwhile emphasizing is the contribution of the reviewers by making available their expertise in different fields along with invaluable insights and recommendations for improving the articles presented in the special issue. We further appreciate additional assistance from the editors-in-chief, Ray E. Patterson and Eric Rolland, to ensure that no conflict of interests exists with respect to our own article by coordinating the associated review process.

\section{References}

1. Cimino MG, Palumbo F, Vaglini G, Ferro E, Celandroni N, La Rosa D (2016) Evaluating the impact of smart technologies on harbor's logistics via BPMN modeling and simulation. Inf Technol Manag. doi:10.1007/s10799-016-0266-4

2. Heilig L, Schwarze S, Voß S (2017) An analysis of digital transformation in the history and future of modern ports. In: Proceedings of the 50th Hawaii international conference on system sciences (HICSS). Big Island, Hawaii

3. Heilig L, Voß S (2016) Information systems in seaports: a categorization and overview. Inf Technol Manag. doi:10.1007/ s10799-016-0269-1

4. Hill A, Böse JW (2016) A decision support system for improved resource planning and truck routing at logistic nodes. Inf Technol Manag. doi:10.1007/s10799-016-0267-3

5. Müller D, Tierney K (2016) Decision support and data visualization for liner shipping fleet repositioning. Inf Technol Manag. doi:10.1007/s10799-016-0259-3

6. Panayides PM, Song DW (2013) Maritime logistics as an emerging discipline. Maritime Policy Manag 40(3):295-308 